\title{
Simulation of the S-Band photon vorticity modulation scheme
}

\author{
Abhay SA ${ }^{1}$, Pavaneeswar BR ${ }^{1}$, Gopinath $\mathbf{S}^{1}$, Sriram BS ${ }^{1}$, Thomas $\mathbf{S J}^{1}$, \\ Shanmugha Sundaram $\mathrm{GA}^{1,2}$ \\ ${ }^{1}$ Department of Electronics and Communications Engineering, Amrita Vishwa Vidyapeetham, Coimbatore 641112, TN, India \\ ${ }^{2}$ Center for Computational Engineering and Networking, Amrita Vishwa Vidyapeetham, Coimbatore 641112, TN, India
}

\section{Email address:}

ga_ssundaram@cb.amrita.edu (G. A. S. Sundaram)

\section{To cite this article:}

Abhay SA, Pavaneeswar BR, Gopinath S, Sriram BS, Thomas SJ, Shanmugha Sundaram GA. Simulation of the S-Band Photon Vorticity Modulation Scheme. American Journal of Electromagnetics and Applications. Vol. 1, No. 2, 2013, pp. 38-43.

doi: 10.11648/j.ajea.20130102.13

\begin{abstract}
Electromagnetic (EM) vorticity modulation is a multi-carrier scheme that offers an enhanced spectral efficiency among the several conventional communication technologies. A mathematical model of the beam-front, carrying different orbital angular momentum (OAM) states, in the S-Band region of the radio frequency (RF) spectrum has been presented using the Laguerre-Gaussian (LG) beam, to describe the effect of RF vorticity and OAM modulation. Results from EM simulations are used to verify the orthogonality of OAM states and thereby highlighting its application in a multiuser environment. Two designs of antenna reflectors are being proposed to generate the orthogonal OAM states $(l=$ 1 and 2). The existence of the OAM states is visualized from the distribution of the current densities and magnetic fields over the aperture area of the reflectors.
\end{abstract}

Keywords: Radio Frequency, Orbital Angular Momentum, Optical Vortices, Electromagnetic Vortices, Parabolic Dish, Spiral Reflector

\section{Introduction}

Radio frequency (RF) spectrum for communication is an exhaustible and hence, now, an expensive resource. Since the invention of the radio by Guglielmo Marconi in 1895, the usage and requirement of spectrum for wireless communication has been ever increasing; and in recent times, due to the scarcity of available bandwidth, the need for reliable and alternative means of communication gained precedence [1]. Various modulation schemes, data congestion techniques, polarization techniques etc. have been implemented so as to provide a solution for the same. One of the recent modulation techniques is orbital angular momentum (OAM) modulation. Theoretically, this technique can provide infinite channels using the same carrier frequency.

Standard electromagnetic (EM) literature describes an EM wave as one that carries not only Linear Momentum but also angular momentum (AM) in the far-field. AM is a combination of spin angular momentum (SAM) and OAM
[2]. The concept of SAM, i.e., polarization of light, has been used since the early 20th century, whereas OAM is a recent addition to the different schemes of modulation [3]. Most of the applications that use OAM modulation have been in the optical domain (where the frequency of operation is in the order of terahertz). Here, we study the utilization of OAM modulation in the RF domain, more specifically, in the SBand region of the spectrum.

In the following section, a mathematical representation of the vortices carrying different OAM states has been discussed and verified that waves carrying different OAM states with the same carrier frequency are orthogonal to each other. Section III discusses the implementation techniques for OAM modulation in the RF domain. The simulation of the experimental setup using a twisted reflector type parabolic dish [1], as well as an $\mathrm{N}$-discrete step parabolic reflector [4] that uses spiral reflector plates, are described in section 4 , followed by the section on results that discuss the distribution of the current density/magnetic fields for either of the designs. 


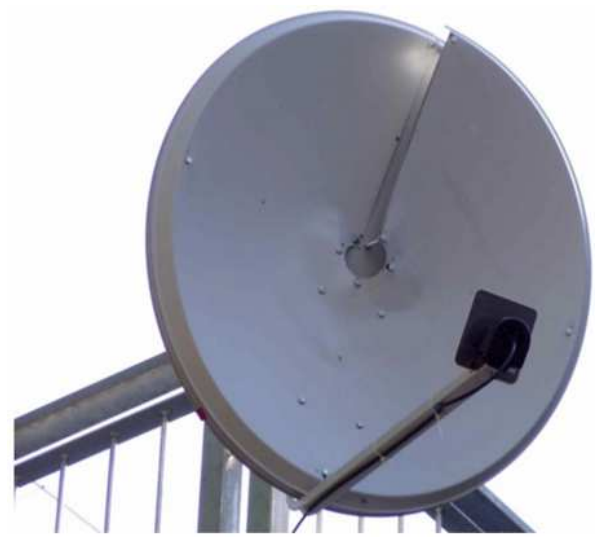

Fig 1: The antenna setup of the experiment performed by F. Tamburini, et al. [1]

\section{Representation Using LG Beams}

Optical vortices are topological defects due to phase singularities in light waves carrying OAM. Any transverse beam of EM radiation can be decomposed into LaguerreGaussian (LG) modes [5]. LG modes are eigen modes of the OAM operator, implying, they can be used as basis functions for representing beams carrying OAM states. This approach assists in representing the wave mathematically.The electric field of an LG beam travelling along z-direction [6], [7] can be expressed in cylindrical coordinates as:

$$
\begin{aligned}
u(r, \phi, z)=\sqrt{\left.\frac{2 p !}{\pi(p}+|l|\right) !} \times \frac{1}{\omega(z)} \times\left[\frac{r \sqrt{2}}{\omega(z)}\right]^{|l|} \\
\quad \times L_{p}^{l}\left(\frac{2 r^{2}}{\omega^{2}(z)}\right) \times e^{\left(\frac{-r^{2}}{\omega^{2}(z)}\right)} \\
\quad \times e^{\left(\frac{-i k r^{2} z}{2\left(z^{2}+z_{R}^{2}\right)}\right)} \\
\quad \times e^{\left(i(2 p+|l|+1) \tan ^{-1}\left(\frac{z}{z_{R}}\right)\right)} \times e^{(-i l \phi)}
\end{aligned}
$$

where, $\omega(z)=\omega_{0} \sqrt{1+\left(\frac{z}{z_{R}}\right)^{2}}, \mathrm{r}$ is the radial distance from the propagation axis, $\phi$ is the azimuthal angle, $\mathrm{z}$ is the propagation distance and $\omega_{0}$ is the zero-order Gaussian radius at the waist; $z_{R}=\pi \frac{\omega_{0}^{2}}{\lambda}$ is the Rayleigh range (with $\lambda$, the wavelength); $k=\frac{2 \pi}{\lambda}$ is the propagation constant; and $L_{p}^{l}(\cdot)$ is the associated Laguerre polynomial, with $p$ and $l$ representing the radial and azimuthal mode numbers, respectively. It can also be seen that the $l^{\text {th }}$ mode of the LG beam has the azimuthal angular dependence of the form $e^{-i l \phi}[6]$. The propagating EM wave consists of $l$ intertwined helical wave fronts, and consequently $l$ is also called the winding number or topological charge [8]. The LG polynomials [9] are given as:

$$
L_{p}^{l}(r)=\frac{e^{r} r^{(-l)}}{p !} \times \frac{d^{p}}{d r^{p}}\left(e^{(-r)} r^{(l+p)}\right)
$$

Using Equation $(1,2)$, the orthogonality of different OAM states has been proved and, the principle of orthogonality [6] is given as follows:

$$
\begin{aligned}
& \left(u_{l 1}(r, \phi, z), u_{l 2}(r, \phi, z)\right) \\
& \triangleq \int u_{l 1}(r, \phi, z) u_{l 2}^{*}(r, \phi, z) r d r d \phi \\
& =\left\{\begin{array}{lr}
0, & \forall l 1 \neq l 2 \\
\int\left|u_{l 1}(r, \phi, z)\right|^{2} r d r d \phi, & l 1=l 2
\end{array}\right.
\end{aligned}
$$

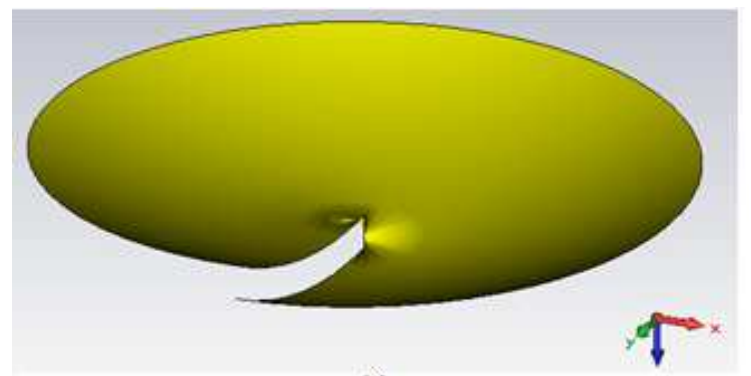

(a)

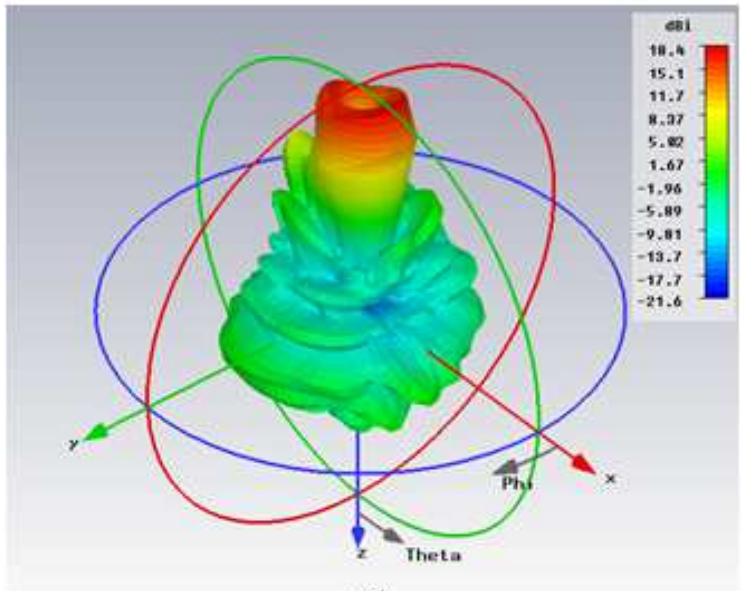

(b)

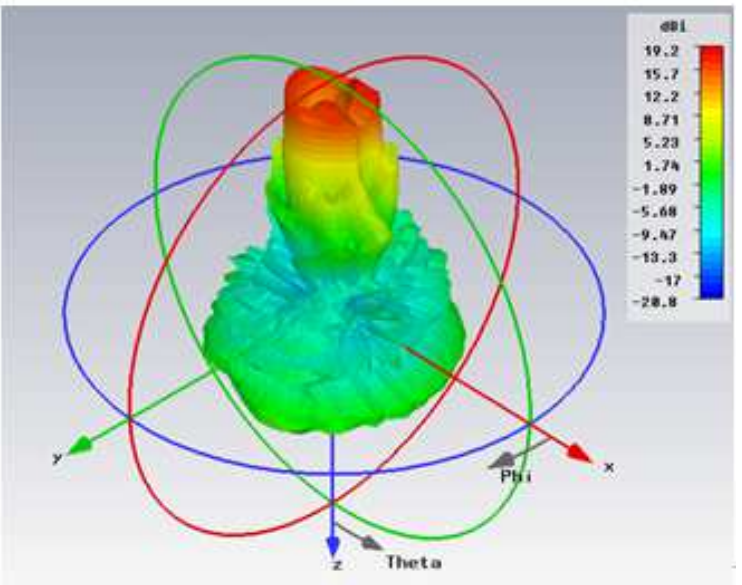

(c)

Fig 2: (a) Twisted parabolic dish; (b) and (c) show radiation patterns obtained using the twisted parabolic dish for OAM state $l=1$ and 2 , respectively. 
where the operator $(0,0)$ denotes the scalar product. This principle holds good for equation (1) and has been verified here using MATLAB simulations.

\section{OAM Modulation in RF Domain}

An experimental setup by F. Tamburini, et.al [1], was one of the first experiments performed using OAM modulation in the RF domain. The antenna design was that of a parabolic dish with a single radial cut introduced on the surface as shown in Fig.1. This design allows different degrees of OAM modulation based on the extent of the twist introduced. This experimental arrangement has been modeled here on the standard microwave studio suite by means of an optimized set of equations, Equations $(5,6,7)$, that was formed from the combination of the equations of a paraboloid and a helix so as to construct a parabolic dish with a similar radial cut as shown in Fig.2(a).
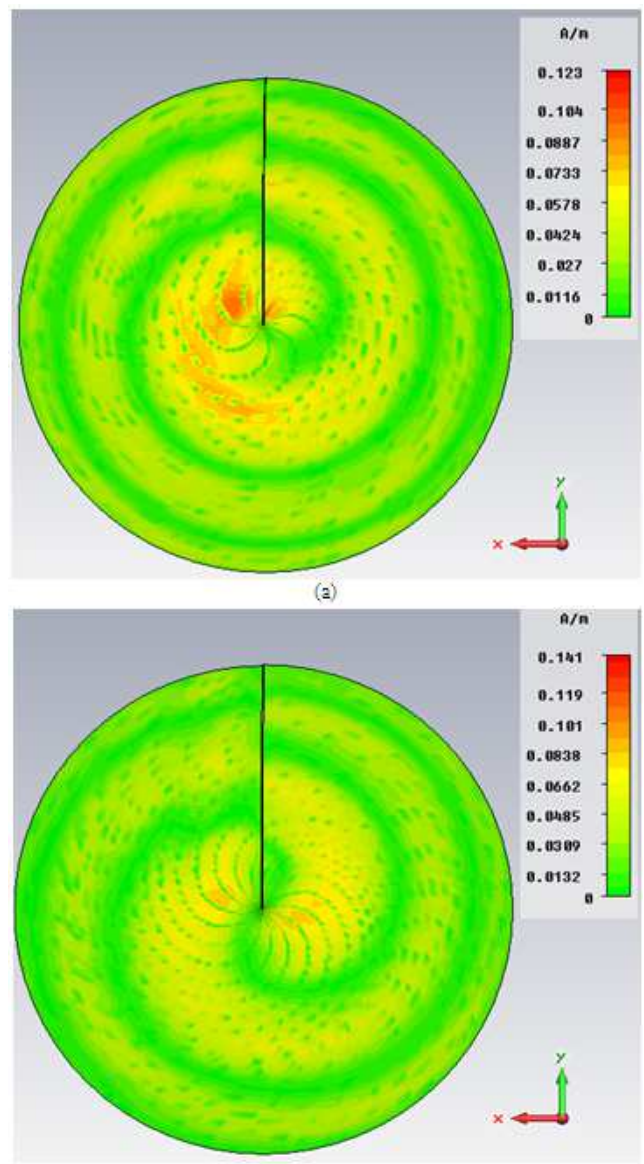

(b)

Fig 3: (a, b) Magnetic field intensity and surface current patterns obtained using the twisted parabolic dish for OAM state $l=1$ and 2, respectively.

Another method of OAM modulation would be the discretization of this dish into $\mathrm{N}$ steps and this has been proposed here. This approach differs from the spiral-phaseplates experiments that were performed in the optical domain [5] and in RF in that while these used planar surfaces with a progressive increase that attains a certain surface pitch across a certain number of discrete sectors; the exercise mentioned here is an implementation of a reflector dish consisting of N-discrete parabolic sectors as can be seen in Fig.4 (a), 5(a), 6(a). This dish can be constructed by introducing $\mathrm{N}$ radial cuts on a normal parabolic dish and giving each of them a progressive "elevation".

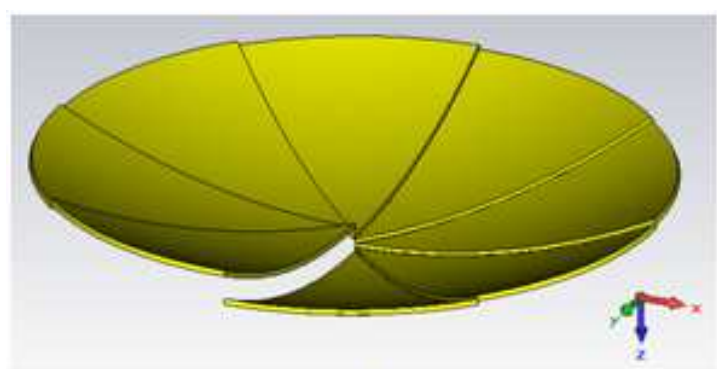

(a)

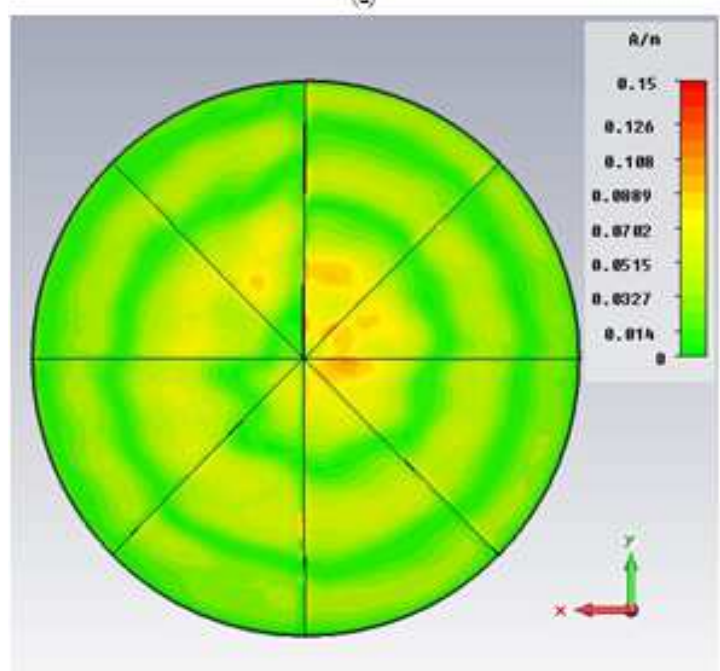

(b)

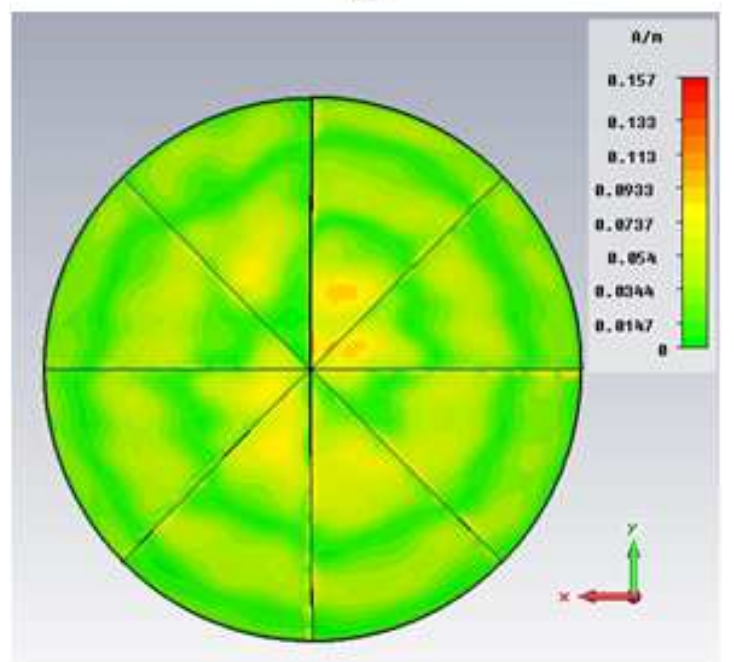

(c)

Fig 4: (a) Twisted parabolic reflector with 8-discrete-step; $(b, c)$ Magnetic field intensity and surface current patterns for 8-discrete-step parabolic reflector designed for $l=1$ and 2 , respectively.

The relation between the OAM state and the carrier wavelength is given by [4]; 


$$
l=\frac{2 h}{\lambda}\left(\frac{N+1}{N}\right)
$$

where $\mathrm{N}$ is the number of discrete steps used in the reflector type antenna, $h$ (surface pitch) represents the height difference between the first and the last plate, similar to the phase gap that was used in the Optical OAM setup [5].

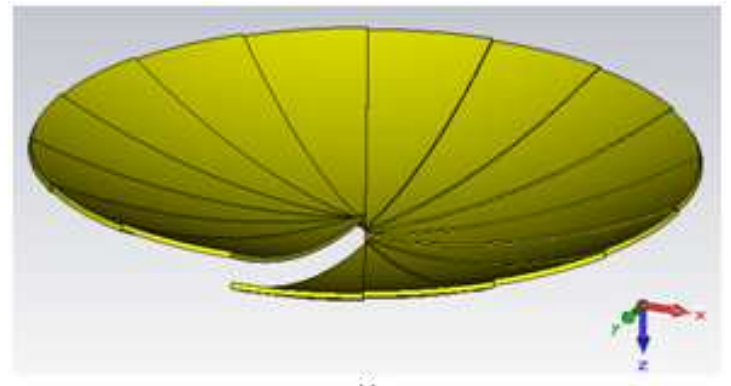

(a)

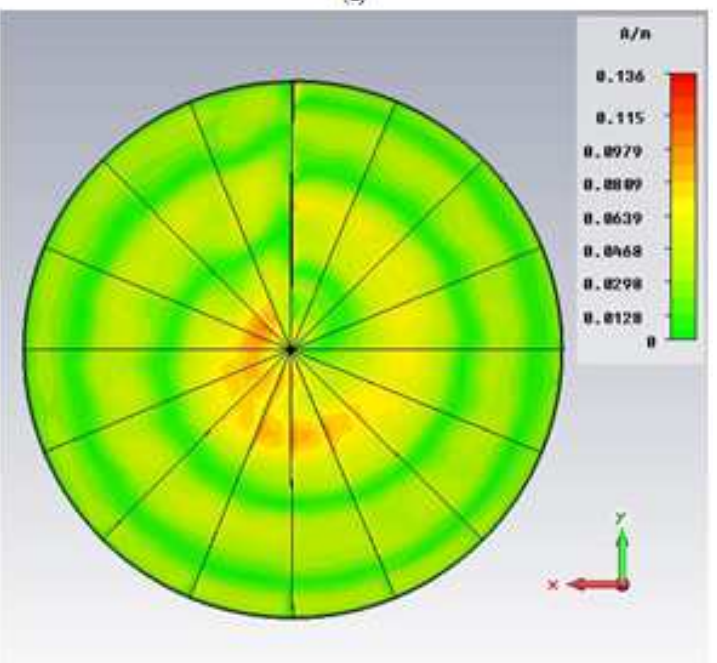

(b)

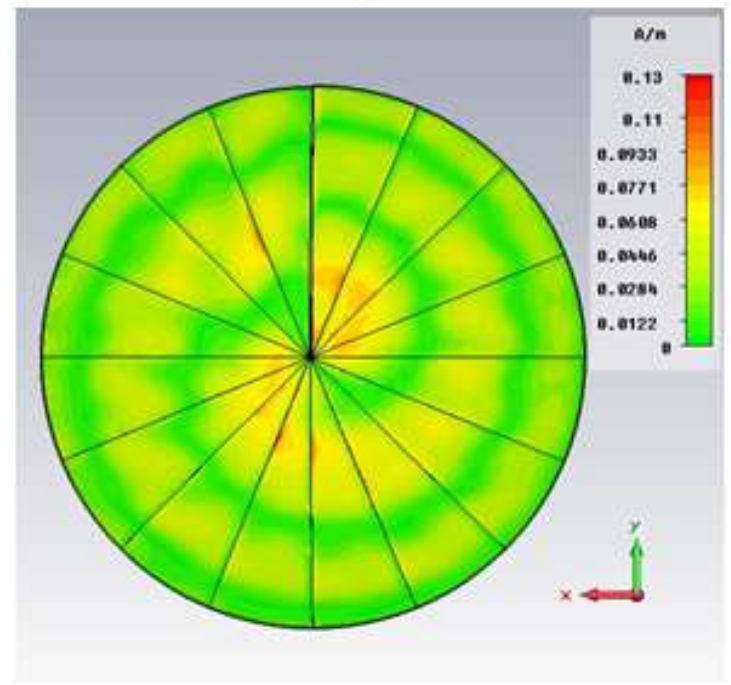

(c)

Fig 5: (a) Twisted parabolic reflector with 16-discrete-step; (b, c) Magnetic field intensity and surface current patterns for 16-discrete-step parabolic reflector designed for $l=1$ and 2, respectively.

As $\mathrm{N}$ increases, the spiral phase plate will reach a more continuous shape since the vertical displacement between adjacent sectors decreases. On allowing $\mathrm{N}$ to reach a sufficiently large number in Equation (4), $l$ approaches an estimated value of $\frac{2 h}{\lambda}$. This forms the basis in implementing a deformed parabolic dish for which values of $l=1$ and 2 have been used along with varying values of $\mathrm{N}$ in the range 1-100 along with a visualization of the radiation patterns in each case.

To prove the aforementioned statement, the parabolic dish with varying $\mathrm{N}$ has been implemented and their radiation patterns are compared.

\section{Simulation}

For the experimental setup [1], a set of equations for the design of the twisted parabolic dish for various OAM states have been formulated whose geometry is shown in Fig. 2(a). Simulation results for this reflector for varying values of 1 and $\mathrm{N}$ are described as follows:

\subsection{Method 1}

For constructing the structure of a twisted reflector in Cartesian coordinates, combination of the equations of a parabola and a helix is used, as mentioned earlier. The proposed set of equations is in Cartesian coordinates define the twisted parabola:

$$
\begin{gathered}
X(u, v)=u * \cos (v), \\
Y(u, v)=u * \sin (v) \\
Z(u, v)=\left(f+\frac{h}{2}\right)-\left(\frac{X(u, v)^{2}+Y(u, v)^{2}}{4 * f}+\frac{v * h}{2 \pi}\right)
\end{gathered}
$$

Here, $u$ and $v$ are the surface variables and vary from 0 to $\mathrm{D} / 2(\mathrm{~cm})$ and 0 to $2 \pi$ (radians) respectively. $\mathrm{D}$ is the diameter of the dish; $f$ defines the focal length of the parabolic dish without the twist. A correction factor of $\mathrm{h} / 2$ has been added to $f$, to account for the twist introduced onto the surface, as the focal point varies with variations to the surface along the positive Z-axis. $h$ defines the surface pitch, i.e. the amount of twist required (that depends on the OAM state and carrier wavelength). This is given as follows:

$$
h=\frac{\lambda * l}{2}
$$

The factor that introduces the twist onto the surface is given by the last term, i.e. $\left(\frac{v}{2 \pi} * h\right)$.

\subsection{Method 2}

The production of various OAM states by the method of an N-discrete step parabolic reflector has also been analyzed. This dish has been modelled in the shape of the regular parabolic dish and divided into N-discrete steps, giving each an equal elevation with respect to the previous plate, thereby obtaining an overall surface pitch $(h)$ as 
mentioned in Equation (4). The angle for each segment is given by $\frac{2 \pi}{N}$. Here:

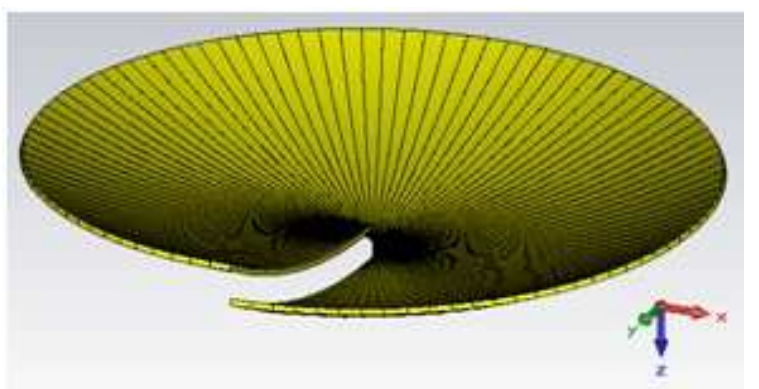

(a)

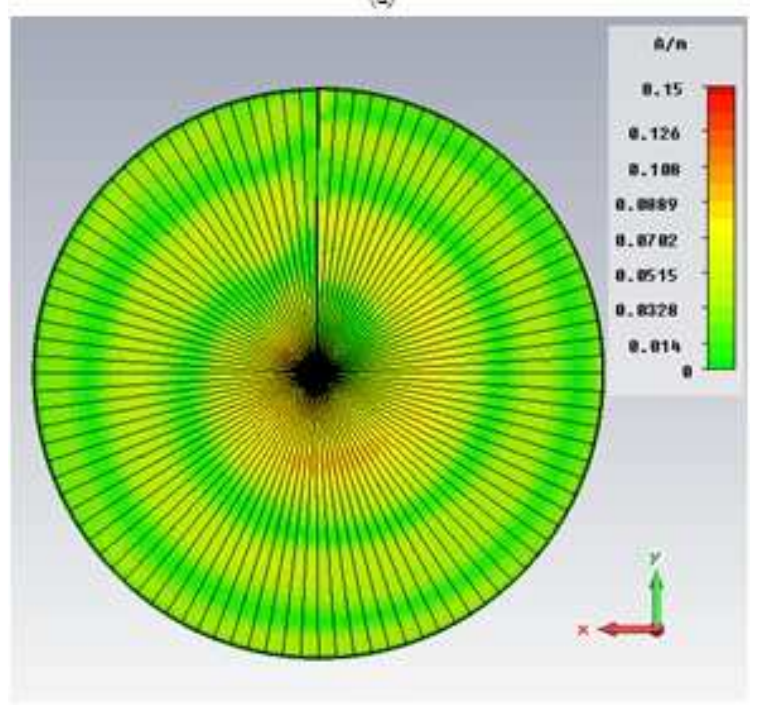

(b)

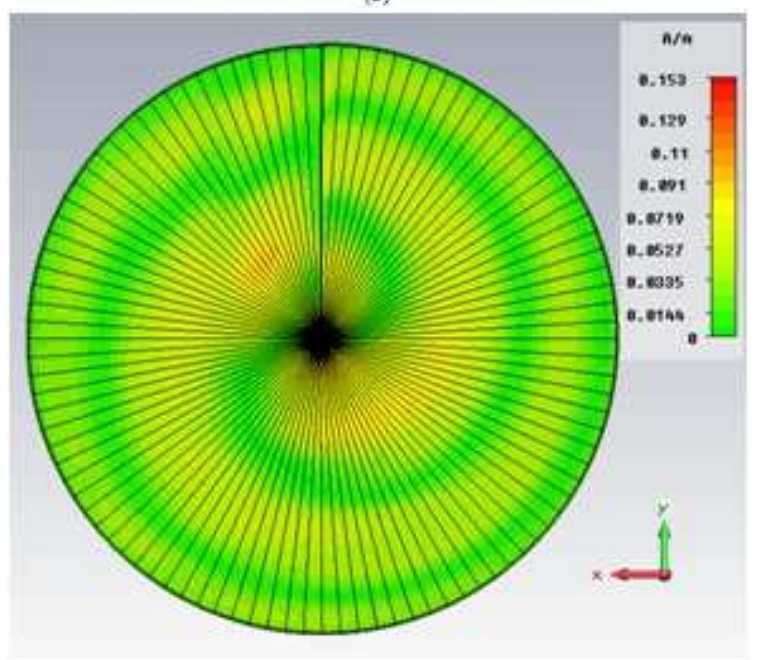

(c)

Fig 6: (a) Twisted parabolic reflector with 100-discrete-step; (b, c) Magnetic field intensity and surface current patterns for a 100-discretestep parabolic reflector design for $l=1$ and 2, respectively.

$$
X(u, v)=u * \cos \left(v+\frac{2 \pi n}{N}\right)
$$

$$
\begin{gathered}
Y(u, v)=u * \sin \left(v+\frac{2 \pi n}{N}\right) \\
Z(u, v)=\left(f+\frac{h}{2}\right)-\left(\frac{X(u, v)^{2}+Y(u, v)^{2}}{4 * f}+\frac{n h}{N}\right)
\end{gathered}
$$

are the surface equations for the design of the discrete parabolic reflector. $n$ varies from 0 to $\mathrm{N}-1$, representing the $\mathrm{N}$ steps of the parabolic dish. $v$ varies from 0 to $\frac{2 \pi}{N}$. The term in Equation (11), $\frac{n h}{N}$, adds the elevation to the $n^{\text {th }}$ step based on the OAM state.

For this simulation activity, $\mathrm{N}$ is chosen as 8, 16 and 100, with higher values of $\mathrm{N}$ providing adequate clarity in terms of visualizing the magnetic field intensity and surface current distributions.

\section{Result}

For simulation purposes, the operational frequency is set in the S-Band at $2.4 \mathrm{GHz}$. The reflector has a diameter, $D=120 \mathrm{~cm}$; a pyramidal horn antenna is used as the feed at the focus $f=0.5 * D$ of the reflector. The material used for the reflector is aluminium and that for the feed is copper.

In this simulation exercise, the choice of $l$ values is observed as being constrained by the degree of deformation to the reflector geometry - large values of $l$ result in unacceptable reflector geometries.

For each of the antenna designs, the 3-D radiation patterns and the magnetic field intensity and surface current distribution over the reflector surface have been plotted.

A surface modification to the reflector design [1] is used to obtain various results for OAM states 1 and 2 .

The magnetic field intensity and surface current distribution plot shows spirals emanating from the centre of the dish. For $l=1$, one spiral can be seen; for $l=2$, two spirals are seen, each being $180^{\circ}$ apart. For the 8-step parabolic reflector, this plot is much distorted and requires an increase in the number of steps to achieve an enhanced result, as can be seen in Figs. 5(c) and 6(c).

The radiation patterns obtained are comparable to those of the twisted parabolic dish used in the first experimental test. The magnetic field intensity and surface current distribution patterns obtained are similar to those of the twisted parabolic dish and confirm the modulation scheme to OAM states 1 and 2, respectively.

\section{Conclusions}

Previous studies on vorticity modulation using the photon OAM concept have established validity for this method as a digital modulation technique across the EM spectrum. This simulation exercise presented here has been conducted by proposing a surface equation for the design of a twisted parabolic dish that was used in a previous experimental setup to demonstrate vorticity modulation. The orthogonality of OAM states, verified mathematically, 
indeed demonstrates a possibility for independent channels that could all use the same carrier frequency. The magnetic field intensity and surface current distribution pattern over the reflector surface design can be used as a visual confirmation to the generation of helical wavefronts that correspond to various OAM states. The approximation for azimuthal mode number $l$, for large $\mathrm{N}$, has also been verified by comparing their radiation patterns.

\section{References}

[1] F. Tamburini, E. Mari, A. Sponselli, B. Thidé, A. Bianchini and F. Romanato, "Encoding many channels on the same frequency through radio vorticity: first experimental test," New Journal of Physics 14 (2012) 033001.

[2] B. Thidé, H. Then, J. Sjöholm, K. Palmer, J. Bergman, T. D. Carozzi, Y. N. Istomin, N. H. Ibragimov, and R. Khamitova, "Utilization of photon orbital angular momentum in the lowfrequency radio domain," Phys. Rev. Lett., vol. 99, no. 8, pp. 087701-1-087701-4, Aug. 2007.

[3] S. M. Mohammadi, L. K. S. Daldorff, J. E. S. Bergman, R. L. Karlsson, B. Thidé, K. Forozesh, T. D. Carozzi, and B. Isham, "Orbital angular momentum in radio-A system study," IEEE Trans. Antennas Propag., vol. 58, no. 2, pp. 565-572, Feb. 2010.

[4] F. Tamburini, E. Mari, B. Thide, C. Barbieri, and F. Romanato, "Experimental verification of photon angular momentum and vorticity with radio techniques." Applied Physics Letters 99, 204102 (2011).

[5] E. Mari, G. Anzolin, F. Tamburini, et al., "Fabrication and testing of $l=2$ optical vortex phase masks for coronography." Opt. Express 18, 2339-2344 (2010).

[6] I. B. Djordjevic, J. A. Anguita, B. Vasic, "Error-Correction Coded Orbital-Angular-Momentum Modulation for FSO Channels Affected by Turbulence," Journal of Lightwave Technology, IEEE 2011.

[7] L.Allen, M. W. Beijerbergen, R. J. C. Spreeuw and J. P. Woerdman, "Orbital angular momentum of light and the transformation of Laguerre-Gaussian laser modes", Physics Review A, Volume 45, Number 11, 1 June, 1992.

[8] Martin Harwit,"Photon Orbital Angular Momentum in Astrophysics." The Astrophysical Journal, 597:1266-1270, 2003 November 10.

[9] F. Pampaloni, J. Enderlein, "Gaussian, Hermite-Gaussian, and Laguerre-Gaussian beams: A primer," arXiv:physics/0410021. 